\title{
Effect of Sodium Hydroxide/Urea/Deionised Water Solution Ratio on the Solubility of Oil Palm Empty Fruit Bunches Extracted Biocellulose
}

\author{
Maha Mohammad AL-Rajabi ${ }^{1}$ and Teow Yeit Haan ${ }^{1,2^{*}}$ \\ ${ }^{I}$ Department of Chemical and Process Engineering, Faculty of Engineering and Built Environment, Universiti \\ Kebangsaan Malaysia, 43600 Bangi, Selangor Darul Ehsan, Malaysia \\ ${ }^{2}$ Research Centre for Sustainable Process Technology (CESPRO), Faculty of Engineering and Built Environment,
}

Universiti Kebangsaan Malaysia, 43600 Bangi, Selangor Darul Ehsan, Malaysia

\begin{abstract}
Biocellulose extracted from oil palm empty fruit bunches (OPEFB) is attracting increased research interest in versatile applications as an alternative material to synthetic cellulose. Normally, biocellulose needs to undergo dissolution prior its applications. Among all explored solvents to dissolve biocellulose, aqueous sodium hydroxide $(\mathrm{NaOH}) /$ urea solution is gaining increased attention. OPEFB biocellulose solubility in $\mathrm{NaOH} /$ urea/deionised (DI) water solution has not been fully studied by researchers. This study aimed to investigate the solubility of OPEFB biocellulose in $\mathrm{NaOH} /$ urea/DI water solution by manipulating the $\mathrm{NaOH} /$ urea/DI water solution ratio and weight percentage of OPEFB biocellulose. Results indicated that increasing the $\mathrm{NaOH} / \mathrm{urea} / \mathrm{DI}$ water solution ratio increased the solubility of OPEFB biocellulose. Further increased $\mathrm{NaOH} / \mathrm{urea} / \mathrm{DI}$ water solution ratio resulted in decreased solubility. Meanwhile, increased OPEFB biocellulose weight percentage decreased the solubility of OPEFB biocellulose in $\mathrm{NaOH} / \mathrm{urea} / \mathrm{DI}$ water solution. The highest solubility of $70.89 \% \pm 1.85 \%$ was exhibited by $7 \% \mathrm{NaOH} / 12 \%$ urea/81\% DI water (w/w) solution and $1 \mathrm{w} / \mathrm{v} \%$ OPEFB biocellulose. This study on OPEFB biocellulose solubility in $\mathrm{NaOH} /$ urea/DI water solution can promote cost-effective and wide utilisation of the abundantly available OPEFB for the synthesis of cellulose fibres, films, and hydrogels in the textile, packaging, and biomedical industries.
\end{abstract}

Keywords: oil palm empty fruit bunches; biocellulose; solubility; sodium hydroxide/urea/deionised water solution

\section{INTRODUCTION}

The increasing palm oil demand has contributed to the fast development of the palm-oil industry in Malaysia (Athirah et al., 2018). This phenomenon has resulted in increased biomass production during palm-oil processing (Teow et al., 2020). One of the major biomass produced from palm-oil processing is oil palm empty fruit bunches (OPEFB). Malaysia is estimated to produce around 7.78 million tonnes of OPEFB annually (Hamzah et al., 2019).

OPEFBs comprise $37 \%-46 \%$ biocellulose, $25 \%-33 \%$ hemicellulose, and 27\%-32\% lignin (Sudiyani et al., 2013).
The conventional practice of burning OPEFBs in boilers as a power source could lead to serious air pollution. Accordingly, awareness on the resources and solid-waste management in the palm-oil industry is raised (Haan et al., 2020). Nazir et al. (2013) successfully extracted a high percentage of biocellulose (64 w/w\%) from OPEFBs, indicating the success of recovering biocellulose from agricultural biomass. The biodegradability, sustainability, high hydrophilicity, and availability of biocellulose in nature render it a favourable natural polymer for various applications, such as ion-battery synthesis (Faizi et al., 2017), biocellulose-membrane 
synthesis for wastewater treatment (Haan et al., 2020), and synthesis of biocellulose hydrogel (Salleh et al., 2019).

Biocellulose needs to undergo dissolution prior to its application. Numerous solvents have been explored to dissolve biocellulose. Among them, aqueous sodium hydroxide $(\mathrm{NaOH}) /$ urea solution is gaining increased attention owing to its simplicity, low toxicity, environment friendliness, and low cost (Yang et. al., 2020; Zainal et al., 2020). However, the solubility of OPEFB biocellulose in $\mathrm{NaOH} /$ urea/deionised (DI) water solution has not yet been fully elucidated. The present study aimed to investigate the solubility of OPEFB biocellulose in $\mathrm{NaOH} /$ urea/DI water solution by adjusting the $\mathrm{NaOH} / \mathrm{urea} / \mathrm{DI}$ water solution ratio and weight percentage of OPEFB biocellulose. The optimum dissolution of biocellulose extracted from the abundantly available OPEFB in the environment-friendly $\mathrm{NaOH} /$ urea/DI water solution is important. It can promote more sustainable and cost-effective applications of OPEFB biocellulose.

\section{MATERIALS AND METHODS}

\section{A. Materials}

OPEFB samples were collected from the Tennamaram palm oil mill in Malaysia. $\mathrm{NaOH}$ and hydrogen peroxide (30 w/w\%) were obtained from Classic Chemicals Sdn. Bhd., Malaysia. Formic acid (98-100, w/w\%) was purchased from Thermo Fisher Scientific, USA. Ethanol (99.5, v/v\%) was supplied by Scienfield Expertise PLT, Malaysia. Urea was purchased from Merck KGaA, Germany.

\section{B. Dissolution of OPEFB Biocellulose}

The biocellulose-extraction process was adapted from the study of Nazir et al. (2013) and modified. OPEFB was initially washed with $1 \mathrm{w} / \mathrm{v} \%$ detergent until the rinse water turned colourless. The washed OPEFB was then dried at $100 \pm 2{ }^{\circ} \mathrm{C}$ until constant weight was obtained. The dried OPEFB was cut, passed through a $1.18 \mathrm{~mm}$-opening mesh sieve, and dewaxed using $70 \mathrm{v} / \mathrm{v} \%$ ethanol in a soxhlet extraction apparatus for 6 $\mathrm{h}$ at $78 \pm 2^{\circ} \mathrm{C}$. The OPEFB fibres were then washed with DI water and dried at $100 \pm 2^{\circ} \mathrm{C}$. $\mathrm{NaOH}$ solution (3 w/v\%) was added to the dewaxed OPEFB fibres, which were heated to $121{ }^{\circ} \mathrm{C}$ for $1 \mathrm{~h}$ for delignification. Acid treatment was then started by soaking $10 \mathrm{~g}$ of delignified OPEFB fibres in $200 \mathrm{~mL}$ of $20 \mathrm{v} / \mathrm{v} \%$ formic acid and $10 \mathrm{v} / \mathrm{v} \%$ hydrogen peroxide mixture. The mixture was heated to $85{ }^{\circ} \mathrm{C}$ for $2 \mathrm{~h}$. Finally, light-yellow OPEFB biocellulose fibres were bleached by suspending in $10 \mathrm{v} / \mathrm{v} \%$ hydrogen peroxide $\left(\mathrm{pH}\right.$ 9) at $60^{\circ} \mathrm{C}$ for $90 \mathrm{~min}$. The OPEFB biocellulose fibres were then rinsed with DI water until neutral $\mathrm{pH}$ was obtained and dried in an oven at $60 \pm 2{ }^{\circ} \mathrm{C}$.

An aqueous solution containing $\mathrm{NaOH} /$ urea/DI water at different weight ratios was prepared as the solvent for OPEFB biocellulose dissolution. Different weight/volume percentages of OPEFB biocellulose was added into $100 \mathrm{~mL}$ of $\mathrm{NaOH} /$ urea/DI water solution and dispersed through continuous stirring at $300 \mathrm{rpm}$ for $5 \mathrm{~min}$. The mixture was then stored under refrigeration at $-12{ }^{\circ} \mathrm{C}$ for $15 \mathrm{~h}$. After thawing the frozen mixture at room temperature and stirred extensively at $1500 \mathrm{rpm}$ for $30 \mathrm{~min}$ (Teow, Ming \& Mohammad, 2018), the mixture was centrifuged at 10000 rpm for $5 \mathrm{~min}$ to remove undissolved OPEFB biocellulose. The dissolved OPEFB biocellulose (supernatant) was separated from the mixture using a pipette. The undissolved OPEFB biocellulose (precipitate) was rinsed several times with DI water until neutral $\mathrm{pH}$ was obtained. Subsequently, the undissolved OPEFB biocellulose was collected, dried in an oven at $60{ }^{\circ} \mathrm{C}$ for $24 \mathrm{~h}$, and weighed. The solubility of OPEFB biocellulose was calculated using Equation (1), and the actual concentration of dissolved OPEFB biocellulose was calculated using Equation (2) (Salleh et al., 2019). The percentages of $\mathrm{NaOH}$, urea, DI water, and OPEFB biocellulose manipulated adjusted in this study were set based on one-factor-at-once and are summarised in Table 1.

Solubility of OPEFB biocellulose (\%) $=\left[\frac{W_{0}-W}{W_{0}}\right] \times 100 \%$

where $\mathrm{W}_{\mathrm{o}}$ was the initial weight of OPEFB biocellulose before dissolution $(\mathrm{g})$, and $\mathrm{W}$ is the weight of undissolved OPEFB biocellulose (g).

Actual concentration of dissolved OPEFB biocellulose $\left(\frac{\mathrm{g}}{\mathrm{mL}}\right)$

$=\frac{\left[\frac{\text { Solubility of OPEFB biocellulose }}{100} \times W_{0}\right]}{V_{S}}$

where $\mathrm{V}_{\mathrm{s}}$ is the volume of $\mathrm{NaOH} / \mathrm{urea} / \mathrm{DI}$ water solution (mL). 
Table 1. Percentage of $\mathrm{NaOH}$, urea, DI water, and OPEFB biocellulose for the dissolution of OPEFB biocellulose

\begin{tabular}{ccccc}
\hline Solution & $\begin{array}{c}\text { NaOH } \\
(\mathbf{w} / \mathbf{w} \%)\end{array}$ & $\begin{array}{c}\text { Urea } \\
(\mathbf{w} / \mathbf{w} \%)\end{array}$ & $\begin{array}{c}\text { DI } \\
\text { water } \\
\text { (w/w\%) }\end{array}$ & $\begin{array}{c}\text { OPEFB } \\
\text { biocellulose } \\
(\mathbf{w} / \mathbf{v} \%)\end{array}$ \\
\hline $\mathrm{SN} 2$ & 2 & 12 & 86 & 1 \\
$\mathrm{SN} 4$ & 4 & 12 & 84 & 1 \\
$\mathrm{SN} 7$ & 7 & 12 & 81 & 1 \\
$\mathrm{SN} 10$ & 10 & 12 & 78 & 1 \\
$\mathrm{SN} 12$ & 12 & 12 & 76 & 1 \\
$\mathrm{SU} 2$ & 7 & 2 & 91 & 1 \\
$\mathrm{SU} 4$ & 7 & 4 & 89 & 1 \\
$\mathrm{SU} 7$ & 7 & 7 & 86 & 1 \\
$\mathrm{SU} 10$ & 7 & 10 & 83 & 1 \\
$\mathrm{SU} 12$ & 7 & 12 & 81 & 1 \\
$\mathrm{SC} 1$ & 7 & 12 & 81 & 1 \\
$\mathrm{SC} 2$ & 7 & 12 & 81 & 2 \\
$\mathrm{SC} 3$ & 7 & 12 & 81 & 3 \\
$\mathrm{SC} 4$ & 7 & 12 & 81 & 4 \\
\hline
\end{tabular}

\section{RESULTS AND DISCUSSION}

\section{A. Effect of $\mathrm{NaOH} /$ Urea/DI Water Ratio}

Figure 1 shows the solubility of OPEFB biocellulose at different $\mathrm{NaOH} /$ urea/DI water ratios. Generally, the solubility of OPEFB biocellulose increased with increased $\mathrm{NaOH}$ and urea percentage in $\mathrm{NaOH} /$ urea/DI water solution. The solubility of OPEFB biocellulose increased with the use of $2 \mathrm{w} / \mathrm{w} \% \mathrm{NaOH}(\mathrm{SN} 2)$ to $7 \mathrm{w} / \mathrm{w} \% \mathrm{NaOH}\left(\mathrm{SN}_{7}\right)$. However, it decreased with further increased $\mathrm{NaOH}$ percentage in $\mathrm{NaOH} /$ urea/DI water solution. This finding was probably due to the concentration-dependent size of $\mathrm{NaOH}-\mathrm{DI}$ water hydrates. At lower $\mathrm{NaOH}$ percentage, $\mathrm{NaOH}-\mathrm{DI}$ water hydrates have large hydrodynamic diameters (Medronho \& Lindman, 2014). Crystalline biocellulose chains were densely packed, with an intersheet distance of about $10 \AA$ and a crystallite diameter of only $5 \mathrm{~nm}$ (Zhang et al., 2010). $\mathrm{NaOH}$ molecules broke the inter- and intra-hydrogen bonds among the biocellulose molecules and destroyed the long-range order of biocellulose crystals to form a biocellulose molecular solution through swelling and activating the biocellulose (Zhang et al., 2010). Thus, at low $\mathrm{NaOH}$ percentage (SN2 and SN4), the hydrodynamic diameter of $\mathrm{NaOH}-\mathrm{DI}$ water hydrates was too large to penetrate the crystalline region of biocellulose, so the solubility of OPEFB biocellulose decreased. Moreover, the amount of $\mathrm{NaOH}$ was insufficient to dissolve biocellulose at low $\mathrm{NaOH}$ percentage (Zhang et al., 2010). Low $\mathrm{NaOH}$ percentage resulted in an insufficient number of alkali ions $\left(\mathrm{Na}^{+}\right)$. The function of $\mathrm{Na}^{+}$was to bring water molecules into biocellulose to cleave hydrogen bonding among biocellulose chains, resulting in biocellulose swelling (Kuo \& Hong, 2005). Consequently, low $\mathrm{NaOH}$ percentage led to poor swelling, thereby decreasing the solubility of OPEFB biocellulose. Among the different ratios of $\mathrm{NaOH} /$ urea/DI water solution, $7 \% \mathrm{NaOH} / 12 \%$ urea/81\% DI water (w/w) solution and $1 \mathrm{w} / \mathrm{v} \%$ OPEFB biocellulose exhibited the highest solubility of $70.89 \% \pm 1.85 \%$. At higher $\mathrm{NaOH}$ percentage (SN1O and $\mathrm{SN} 12$ ), the concentration of $\mathrm{Na}^{+}$ was too high, so the hydration of $\mathrm{Na}^{+}$was low because of the insufficient water molecules (Kuo \& Hong, 2005). The hydration of the low amount of ions decreased the frequency of hydrogen-bonding cleaving, resulting in poor swelling of biocellulose. Thus, the solubility of the OPEFB biocellulose decreased.

Meanwhile, OPEFB biocellulose solubility increased with increased urea percentage in $\mathrm{NaOH} /$ urea/DI water solution from $2 \mathrm{w} / \mathrm{w} \%$ urea (SU2) to $12 \mathrm{w} / \mathrm{w} \%$ urea (SU12). The dissolution of biocellulose in $\mathrm{NaOH} /$ urea/DI water solution occurred through the formation of a biocellulose- $\mathrm{NaOH}-$ urea inclusion complex (IC), where $\mathrm{NaOH}$ and urea surrounded the biocellulose chains in a hydrate layer to form a wormlike structure (Huber et al., 2016). Urea in biocellulose- $\mathrm{NaOH}$-urea aqueous solution existed in two forms, namely, hydrates associated with the IC and free hydrates. Both forms existed in equilibrium with each another. A higher percentage of urea resulted in excess urea free hydrates, which displaced the equilibrium toward the inclusion of urea in IC, thereby strengthening the shielding of biocellulose chains (Huber et al., 2016). The formation of IC minimised the intermolecular biocellulose interactions, leading to increased OPEFB biocellulose stability. 


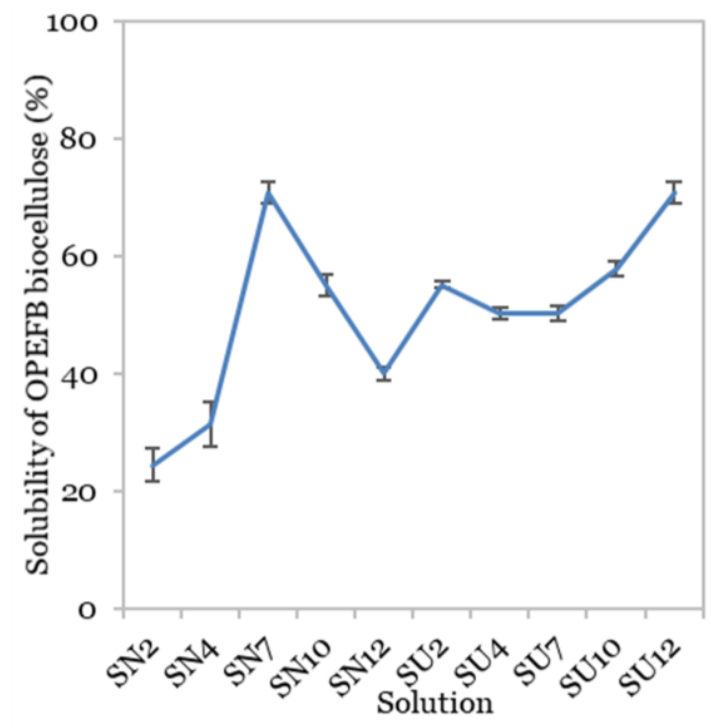

Figure 1. Solubility of OPEFB biocellulose at different $\mathrm{NaOH} /$ urea/DI water ratios

\section{B. Effect of OPEFB Biocellulose Weight Ratio}

Given that $7 \% \mathrm{NaOH} / 12 \%$ urea/81\% DI water $(\mathrm{w} / \mathrm{w})$ exhibited the highest OPEFB biocellulose solubility of $70.89 \% \pm 1.85 \%$, effect of OPEFB biocellulose weight ratio was studied at this constant $\mathrm{NaOH} /$ urea/DI water ratio. Figure 2 shows the solubility of OPEFB biocellulose and actual concentration of dissolved OPEFB biocellulose at different OPEFB biocellulose weight ratios. The solubility of OPEFB biocellulose decreased with increased initial OPEFB biocellulose percentage in $\mathrm{NaOH} / \mathrm{urea} / \mathrm{DI}$ water solution. Low-percentage OPEFB biocellulose in $\mathrm{NaOH} /$ urea/DI water solution was more efficient for mixing as it facilitated the contact between $\mathrm{NaOH} /$ urea/DI water solution and OPEFB biocellulose, thereby allowing a higher percentage of OPEFB biocellulose to dissolve. Additionally, the self-aggregation of OPEFB biocellulose at a high initial percentage of OPEFB biocellulose may have caused the low solubility. This observation has been made by Kaco et al. (2014), who found that the solubility of biocellulose derived from kenaf core powder in $\mathrm{NaOH} /$ urea/DI water solution decreases with increased biocellulose weight percentage.

Meanwhile, the actual concentration of dissolved OPEFB biocellulose increased with increased initial OPEFB biocellulose percentage from $1 \mathrm{w} / \mathrm{v} \%$ to $3 \mathrm{w} / \mathrm{v} \%$ and achieved a plateau after $3 \mathrm{w} / \mathrm{v} \%$. This finding could be due to the limited capacity of $\mathrm{NaOH} / \mathrm{urea} / \mathrm{DI}$ water solution for dissolving cellulose. When the maximum dissolution capacity of $\mathrm{NaOH} /$ urea/DI water solution was reached, the excess OPEFB biocellulose added into $\mathrm{NaOH} /$ urea/DI water solution did not dissolve, causing a plateau trend for the actual concentration of dissolved OPEFB biocellulose.

OPEFB biocellulose was not completely dissolved in $\mathrm{NaOH} /$ urea/DI water solution possibly because of the high molecular weight and degree of polymerisation (DP) of OPEFB biocellulose. This feature can affect the penetration of $\mathrm{NaOH} /$ urea/DI water solution into OPEFB biocellulose resulting in partial solubility and producing undissolved OPEFB biocellulose (Baharin et al., 2018). The high molecular weight of OPEFB biocellulose molecules corresponds with a large excluded volume that promotes intermolecular interaction and thus suppresses its solubility (Guo et al., 2017). Conversely, a high DP resulted in a network of fibres held together through hydrogen bonds forming longchain segments. Thus, OPEFB biocellulose with high DP was strong and resisted dissolution (Ni et al., 2015). The solubility of OPEFB biocellulose ranging from $44.12 \% \pm 3.03 \%$ to $70.89 \% \pm 1.85 \%$ has been found to be higher than that of OPEFB biocellulose at $54.52 \% \pm 0.50 \%$ (Salleh et al., 2019). Salleh et al. (2019) used OPEFB cellulose and 7\% NaOH/12\% urea/81\% DI water (w/w) solvents at $-12{ }^{\circ} \mathrm{C}$ refrigeration, which is comparable to the solvent and temperature in the current study. Our higher OPEFB-biocellulose solubility than that in literature supported the successful dissolution of the maximum percentage of OPEFB biocellulose in $\mathrm{NaOH} /$ urea/DI water solution in the current study.

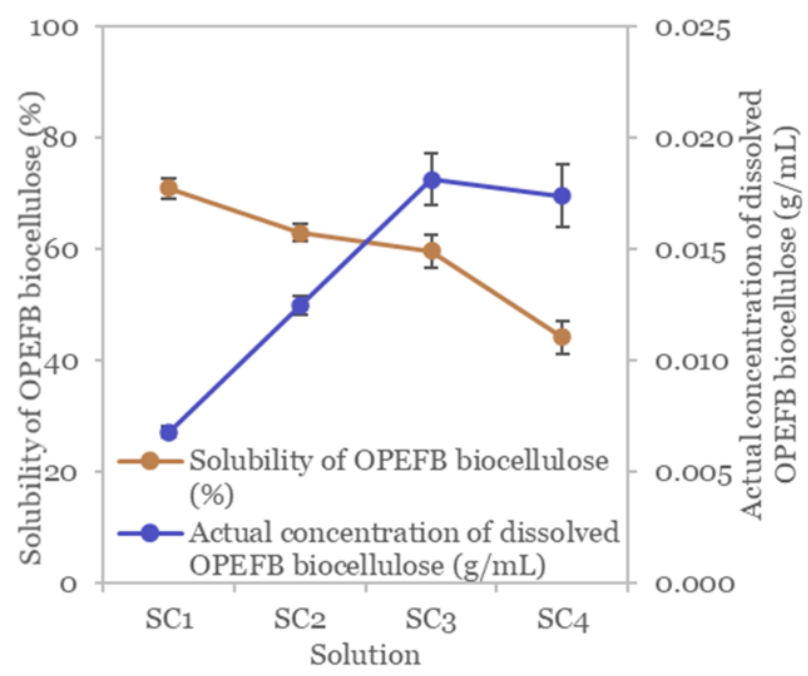

Figure 2. Solubility of OPEFB biocellulose and actual concentration of dissolved OPEFB biocellulose at different OPEFB biocellulose weight ratios 


\section{CONCLUSION}

The dissolution of OPEFB biocellulose in different $\mathrm{NaOH} /$ urea/DI water solution ratios and weight percentages of OPEFB biocellulose was investigated. At different $\mathrm{NaOH} /$ urea/DI water solution ratios, $7 \% \mathrm{NaOH} / 12 \%$ urea/ $81 \%$ DI water $(\mathrm{w} / \mathrm{w})$ solution and $1 \mathrm{w} / \mathrm{v} \%$ OPEFB biocellulose exhibited the highest solubility of $70.89 \% \pm 1.85 \%$. However, OPEFB biocellulose solubility decreased at higher $\mathrm{NaOH}$ percentage (SN10 and SN12). Meanwhile, the solubility of OPEFB biocellulose decreased with increased initial OPEFB biocellulose percentage in $\mathrm{NaOH} / \mathrm{urea} / \mathrm{DI}$ water solution. The low-percentage OPEFB biocellulose in $\mathrm{NaOH} /$ urea/DI water solution was more efficient for mixing and facilitated the contact between $\mathrm{NaOH} /$ urea/DI water solution and
OPEFB biocellulose, thereby allowing a higher percentage of OPEFB biocellulose to be dissolved. The optimum dissolution of $1 \mathrm{w} / \mathrm{v} \%$ biocellulose extracted from the abundantly available OPEFB in the environment-friendly $7 \% \mathrm{NaOH} / 12 \%$ urea/81\% DI water $(\mathrm{w} / \mathrm{w})$ solution can promote more sustainable and cost-effective applications of OPEFB biocellulose. Further study on the solubility of OPEFB biocellulose using different solvents is suggested.

\section{ACKNOWLEDGEMENT}

The authors gratefully acknowledge the funding for this work by Dana Modal Insan (MI-2019-017) and Dana Padanan Kolaborasi (DPK-2021-002).

\section{REFERENCES}

Athirah, WN, Haan, TY \& Mohammad, AW 2018, 'Sustainable approach in Palm Oil Industry-Green Synthesis of Palm Oil Mill Effluent Based Graphene Sand Composite (P-GSC) for Aerobic Palm oil mill effluent treatment', Jurnal Kejuruteraan SI, vol. 1, no. 7, pp. 11-20. doi: 10.17576/jkukm-2018-si1.

Baharin, KW, Zakaria, S, Ellis, AV, Talip, N, Kaco, H, Sinyee, G, Zailan, FD \& Hashim, SNASH 2018, 'Factors affecting cellulose dissolution of oil palm empty fruit bunch and kenaf pulp in $\mathrm{NaOH} /$ urea solvent', Sains Malaysiana, vol. 7 , no. 2, pp. 377-386. doi: 10.17576/jsm-2018-4702-20.

Faizi, MK, Shahriman, AB, Majid, MA, Shamsul, BMT, Ng, YG, Basah, SN, Cheng, EM, Afendi, M, Zuradzman, MR, Wan, K \& Hazry, D 2017, 'An overview of the Oil Palm Empty Fruit Bunch (OPEFB) potential as reinforcing fibre in polymer composite for energy absorption applications', in MATEC Web of Conferences.

Guo, MQ, Hu, X, Wang, C \& Ai, L 2017, 'Polysaccharides: structure and solubility', in Solubility of Polysaccharides, ntechOpen, pp. 1-17. doi: 10.5772/intechopen.71570.

Huber, T, Starling, K, Cen, WS, Fee, C \& Dimartino, S 2016, 'Effect of urea concentration on the viscosity and thermal stability of aqueous $\mathrm{NaOH} /$ urea cellulose solutions', Journal of Polymers, 2016.

Kaco, H, Zakaria, S, Razali, NF, Chia, CH, Zhang, L \& Jani, SM 2014, 'Properties of cellulose hydrogel from kenaf core prepared via pre-cooled dissolving method', Sains
Malaysiana, vol. 43, no. 8, pp. 1221-1229.

Kuo, Y \& Hong, J 2005, 'Investigation of solubility of microcrystalline cellulose in aqueous $\mathrm{NaOH}^{\prime}$, Polymers for Advanced Technologies, vol. 16, pp. 425-428. doi: 10.1002/pat.595.

Medronho, B \& Lindman, B 2014, 'Brief overview on cellulose dissolution/regeneration interactions and mechanisms', Advances in Colloid and Interface Science, vol. 222, pp. 502-508. doi: 10.1016/j.cis.2014.05.004.

Nazir, MS, Wahjoedi, BA, Yussof, AW \& Abdullah, MA 2013, 'Eco-friendly extraction and characterization of cellulose from oil palm empty fruit bunches', BioResources, vol. 8, no. 2, pp. 2161-2172. doi: 10.15376/biores.8.2.2161-2172.

Ni, J, Teng, N, Chen, H, Wang, J, Zhu, J \& Na, H 2015, 'Hydrolysis behavior of regenerated celluloses with different degree of polymerization under microwave radiation', Bioresource Technology, vol. 191, pp. 229-233. doi: 10.1016/j.biortech.2015.05.036.

Salleh, KM, Zakaria, S, Sajab, MS, Gan, S \& Kaco, H 2019, 'Superabsorbent hydrogel from oil palm empty fruit bunch cellulose and sodium carboxymethylcellulose', International Journal of Biological Macromolecules, vol. 131, pp. 50-59. doi: 10.1016/j.ijbiomac.2019.03.028.

Teow, YH, Tajudin, SA, Ho, KC \& Mohammad, AW 2020, 'Synthesis and characterization of graphene shell composite from oil palm frond juice for the treatment of dyecontaining wastewater', Journal of Water Process 
Engineering, vol. 35(2020). doi: 10.1016/J.JWPE.2020.101185.

Teow, Y, Ming, K \& Mohammad, A 2018, 'Synthesis of cellulose hydrogel for copper (II) ions adsorption', Journal of Environmental Chemical Engineering, vol. 6, no. 4, pp. 4588-4597. doi: 10.1016/j.jece.2018.07.010.

Yang, J, Medronho, B, Lindman, B \& Norgren, M 2020, 'Simple one pot preparation of chemical hydrogels from cellulose dissolved in cold LiOH/Urea', Polymers, vol. 12, no. 2. doi: 10.3390/polym12020373.

Zainal, SH, Mohd, NH, Suhaili, N, Anuar, FH, Lazim, AM \& Othaman, R 2020, 'Preparation of cellulose-based hydrogel: a review', Journal of Materials Research and Technology. doi: 10.1016/j.jmrt.2020.12.012.

Zhang, S, Li, FX, Yu, JY \& Hsieh, YL 2010, 'Dissolution behaviour and solubility of cellulose in $\mathrm{NaOH}$ complex solution', Carbohydrate Polymers, vol. 81, no. 3, pp. 668674. doi: 10.1016/j.carbpol.2010.03.029. 